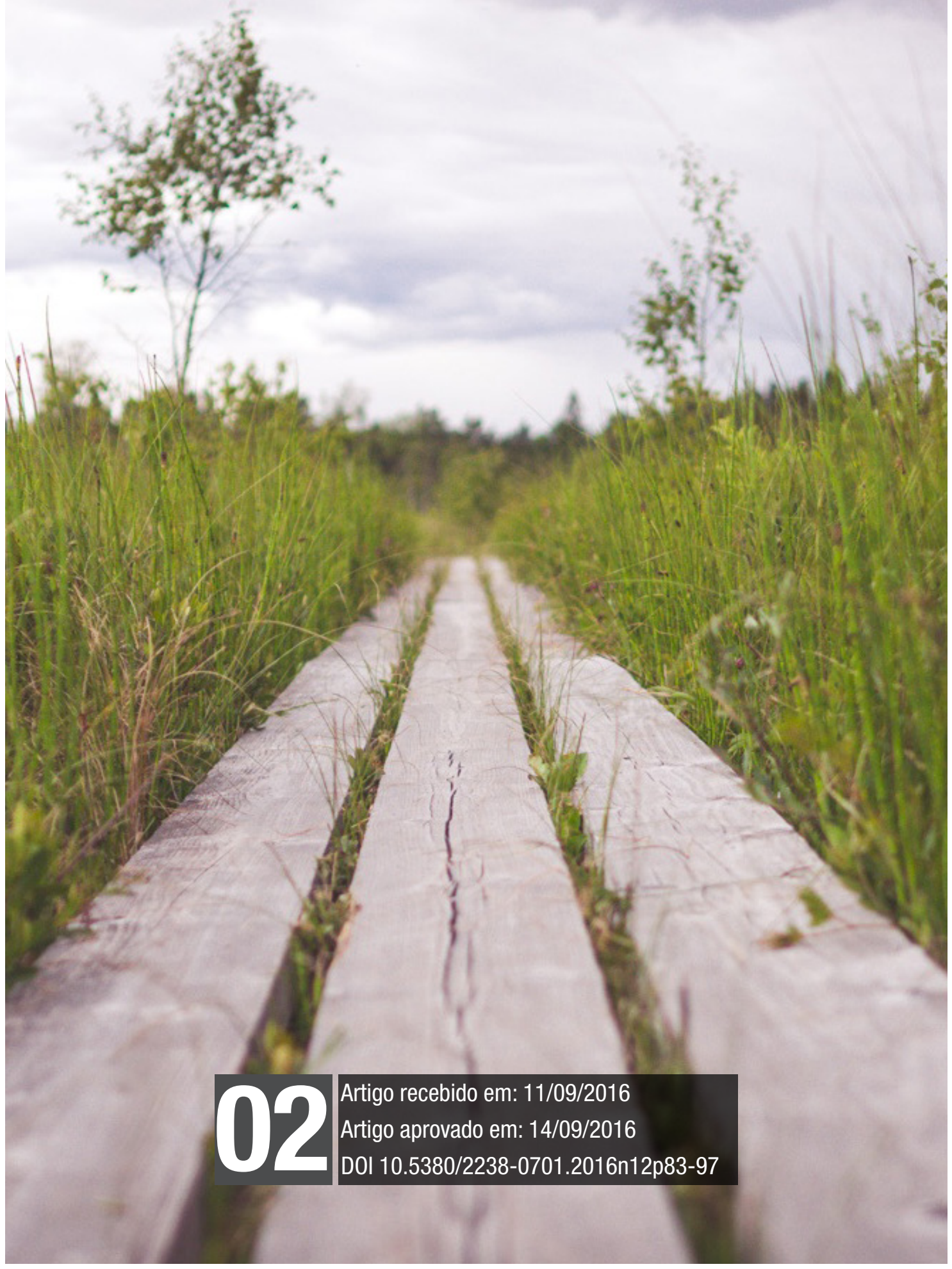


Pesquisa-ação. Dispositivo. Engenharia cooperativa.

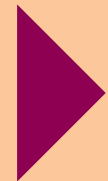




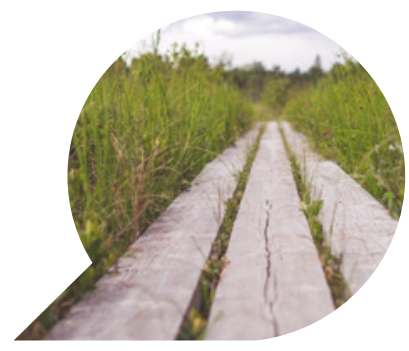

\title{
Um regresso à noção de abordagem dispositivo?
}

\author{
Un retour en actualité de la notion \\ de démarche dispositive?
}

Is the "systems-like" approach back in research?

\section{GENEVIÈVE LAMEUL ${ }^{2}$}

Resumo: $\mathrm{O}$ artigo destaca a noção de démarche dispositive que Geneviève Jacquinot desenvolveu com Hugues Choplin em 2002. Esta noção, articulando dispositivo e inovação, posiciona-se em relação a outras formas de pesquisa, mais lembradas hoje, como as engenharias cooperativas ou design based recherch. Escrito de maneira bem pessoal, a partir da experiência da autora, o artigo questiona a cumulatividade dos resultados de pesquisa assim como as ligações e pontes a construir entre elas, de modo a acessar melhor a inteligibilidade das situações sociais cada vez mais complexas. Esclarecendo estas diferentes situações, onde a pesquisa e a ação se entrecruzam e se esclarecem mutualmente, as posições dos autores correspondendo a cada configuração também serão questionadas.

Palavras-chave: Pesquisa-ação; Dispositivo; Démarche dispositive; Design Based Recherch; Engenharia Cooperativa.

1 Tradução de Rosa Maria Cardoso Dalla Costa.

2 Université Rennes 2, França, genevieve.lameul@uhb.fr 
Résumé: L'article remet en lumière la notion de démarche dispositive que Geneviève Jacquinot a développée avec Hugues Choplin en 2002. Cette notion, à l'articulation entre dispositif et innovation, est positionnée par rapport à d'autres formes de recherche, plus souvent évoquées aujourd'hui, comme les ingénieries coopératives ou le design based research. Écrit de manière très personnelle, à partir de l'expérience de l'auteure, l'article interroge la cumulativité des résultats de recherche ainsi que les liens et ponts à construire entre eux pour accéder à une meilleure intelligibilité des situations sociales de plus en plus complexes. Précisant ces différentes situations où recherche et action s'entrecroisent et séclairent mutuellement, les postures des acteurs correspondant à chaque configuration se trouvent aussi questionnées.

Mots-clés: recherche action, dispositif, démarche dispositive, design based research, ingénierie coopérative.

Abstract: This paper highlights the concept of systemic approach developed by Geneviève Jacquinot and Hugues Choplin in 2002. This concept, at the crossroads between innovation and computing systems can be seen in the context of other forms of research most often considered today as cooperative engineering or design based research. Written from a very personal point of view based on the experience of the author, the article questions the cumulativity of research results and also the links and bridges to build to access better understandability of increasingly complex situations. It specifies the different situations where research and action are present and intersect and enlighten each other, and questions the postures of the actors in each configuration.

Keywords: action research, systems, innovation, systemic approach, design based research, collaborative engineering. 


\section{Introdução}

Este artigo segue o modelo dos escritos narrativos pessoais. Falar da influência dos trabalhos de Geneviève Jacquinot sobre meu percurso de pesquisadora tem como finalidade testemunhar meu reconhecimento e, ao mesmo tempo, ilustrar o acúmulo de resultados de pesquisa em ciências humanas e sociais. Nesta perspectiva, ainda é necessário destacar a abertura criativa e o diálogo interdisciplinar que consegue instalar uma caminhada inovadora para melhorar a qualidade da pesquisa relativa aos dispositivos inovadores.

\section{Contextualização}

Nos anos 2000, Geneviève Jacquinot propôs com Hugues Choplin a noção de démarche dispositive para caracterizar um elemento de método de pesquisa inovador ${ }^{3}$. Esta noção foi confrontada com a prática para descrever os dispostivos pedagógicas inovadores, como os campos virtuais, sobretudo na universidade online (UEL). Se o seu surgimento já podia ser pressentido num número especial a respeito da noção de dispositivo (JACQUINOT-DELAUNAY; MONNOYER, 1999), a meu ver, foram uma obra (JACQUINOT; FICHEZ, 2008) e um artigo (JACQUINOT; CHOPLIN, 2002) os que melhor aprofundaram este conceito e o tornaram conhecido. $\mathrm{Na}$ minha caminhada profissional como engenheira de formação, esta noção ecoou junto com a noção de "engenharia cooperativa" empregada por Brigitte Gruson, por ocasião da defesa de sua tese de doutorado no centro de pesquisa sobre a educação, aprendizados e a didática (CREAD) ${ }^{4}$. Em seguida, foi no contexto da pesquisa coletiva HY-SUP (2009-13) que, graças a Bernadette Charlier, pude enten-

3 A pesquisa inovação caracteriza-se por dois outros elementos: i) trata-se de uma pesquisa com finalidade prática (praxéologique), menos no sentido clássico do termo que no sentido mais contemporâneo, desenvolvido por B. Albéro e C. Brassac (2003) sobre a noção de "práxis do conhecimento"; ii) inter ou transdisciplinar, seus objetos constroem-se no entroncamento de diversas disciplinas, o que implica uma análise tanto dos processos da ação como dos conhecimentos, afim de sustentar seu desenvolvimento.

4 Gruson, B. (2006). Tese defendida na Universidade de Rennes 2. “L'enseignement d'une langue étrangère à l'école et au collège: vers une meilleure compréhension des situations didactiques mises en oeuvre: analyse comparative de l'action de deux professeurs de CM2 et de deux professeurs de sixième". "O ensino de uma língua estrangeira na escola e no colégio: em direção a uma melhor compreensão das situações didáticas colocadas em prática: análise comparativa da ação de dois professores de CM2 e de dois professores de sexto ano". http://catalogue.bu.univ-rennes2.fr/cgibin/koha/opac-detail.pl?biblionumber=348105 
der o funcionamento da design based research. Esta se tornou outra oportunidade de me lembrar da noção de "démarche dispositive". De forma complementar, na unidade de pesquisa onde faço meus trabalhos (CREAD, EA 3875), a leitura das publicações de meus colegas -sobretudo os de Brigitte Albero (2009, 2010); Jérome Eneau (2011) e Jérome Guérin, (2012) - permitiu-me aprofundar a noção de dispositivo e sensibilizou-me para outras formas de pesquisa diferentes das que eu tinha feito na tese.

Esta faísca de reencontros humanos e intelectuais conduziu-me a me interessar, particularmente, pelos fins epistemológicos e transformadores produzidos por uma articulação "razoável" entre a pesquisa e a ação. É um dos temas que comecei a desenvolver com Catherine Loisy e os co-autores da obra que acabamos de organizar (LAMEUL; LOISY, 2014) questionando a maneira como a pesquisa e a ação podem se articular para uma melhor compreensão das situações pedagógicas que utilizam a informática. A redação deste artigo faz parte de meu projeto atual de um estudo aprofundado desta variedade de maneiras de intervir sobre o mundo e de sua complementaridade para construir um outra realidade mais inteligível.

Neste artigo, me preocuparei, numa primeira parte, em definir e situar abordagens e noções reencontradas ao longo de meu percurso que parecem ter um ar familiar com a noção de démarche dispositive. Na segunda parte, retomo a definição proposta por Geneviève Jacquinot da "démarche dispositive", noção mais importante desta contribuição. Concluirei, numa terceira parte, fazendo dois tipos de perguntas ao leitor: questionarei a partir deste exemplo a maneira como as noções se posicionam umas em relação às outras no campo da atividade e da pesquisa em ciências humanas e sociais; e também interrogarei se continua atual a noção de démarche dispositive num mundo engajado na corrida da inovação. Enfim, concluirei sobre as evoluções de posturas que uma tal caminhada implica, tanto entre os pesquisadores como entre os profissionais.

\section{Panorama das diferentes formas de pesquisa}

No ensino superior, opera-se um duplo movimento: uma forte incitação social e política à inovação conduz a criar situações onde a mudança de práticas se realiza, mesmo ao preço de um número 
elevado de incertezas. A tendência está em voltar-se em direção à pesquisa em ciências humanas e sociais para estudar e esclarecer estas situações complexas. Impõe-se, então, uma revisão profunda dos métodos de pesquisa que parece orientar-se em direção a uma maior tomada de consciência da dimensão humana assim como da atividade real do sujeito (REMOUSSENARD, 2005). As interações entre o homem e seu ambiente devem ser estudadas dentro da dinâmica da ação. A fronteira entre os pesquisadores e os profissionais em ação, tende a romper-se, pois nasce progressivamente uma maior consciência que é de seu diálogo que vai surgir uma inteligibilidade maior das situações. E é no contexto geral da evolução social e científica que se manifestam diferentes iniciativas participando mais ou menos desta aproximação. Vamos dar uma visão rápida destas visões entre elas e situar a démarche dispositive em relação a estas últimas.

\section{O design based research}

Os métodos de pequisa envolvidos na visão da design-based research (VAN DEN AKKER; GRAVEMEIJER; MDKENNEY; NIEVEEN, 2006) que começam a ser divulgados, contribuem para conhecer estas novas modalidade de colaboração entre pesquisadores e profissionais. As principais característas da design-based research (trabalho participativo, cooperação, interação, centrada sobre o processo, a utilidade e a teoria) visam criar as condições favoráveis a este diálogo entre pesquisadores e profissionais que nos parece cada vez mais indispensável.

$\mathrm{Na}$ sua grande ambição de estudar os dispositivos híbridos de formação no ensino superior, a pesquisa HY-SUP (2009-13) confrontou os pesquisadores com a complexidade e a diversificação das práticas de ensino. Como testemunham os resultados do dispositivo de avaliação colocado em prática, ela lhes permitiu aprender desta experiência e tomar conhecimento que a adoção de uma análise de tipo design based research, aplicada em situações precisas, impunha-se provavelmente como um método mais adequado para chegar a uma compreensão fina e duradoura dos impactos de um dispositivo híbrido de formação sobre o desenvolvimento profissional dos professores, sobre o aprendizado dos estudantes e sobre a evolução da instituição. A avaliação do processo de trabalho metodológico dos membros da equipe HY-SUP (DESCHRYVER; CHARLIER, 2013, p. 252) valoriza 
esta aproximação para a sequência de seus trabalhos e se recomenda às equipes que estão envolvidas com este mesmo tipo de pesquisa complexa. A tabela abaixo reagrupa, de maneira sintética, as grandes características deste método que sustentam esta proposição.

Tabela 1: Características do design based research a partir de uma tradução livre de Van den Akker et al. (2006, p.5).

\begin{tabular}{|l|l|}
\hline Características & O método da design based research... \\
\hline Dimensão intervencionista & $\ldots$ tem por finalidade intervir em situações reais \\
\hline Forma interativa & $\begin{array}{l}\ldots \text { adota uma aproximação interativa de concepção, ava- } \\
\text { liação, revisão }\end{array}$ \\
\hline Orientação em direção ao processo & $\begin{array}{l}\ldots \text { tem por objetivo compreender e melhorar a interven- } \\
\text { ção }\end{array}$ \\
\hline Orientação para a utilidade & $\begin{array}{l}\ldots \text { tem como preocupação avaliar o impacto sobre e para } \\
\text { as pessoas e as instituições }\end{array}$ \\
\hline Orientação para a teoria & $\begin{array}{l}\text { b. está baseada sobre as proposições teóricas que contri- } \\
\text { buem para a construção de conhecimentos }\end{array}$ \\
\hline
\end{tabular}

Concretamente, trata-se de proceder "a estudos de caso quase experimentais inscritos ao longo do tempo" (CRAHAY, 2006, p. 49), isto é, a propósito de um dispositivo e com a colaboração do ou dos professores envolvidos, de realizar coletas de dados objetivos e subjetivos antes de toda intervenção (linha de base), de escolher uma intervenção em função dos objetivos fixados pelo ensino, de realizar novas coletas de dados ao longo da intervenção, eventualmente de retirar a intervenção ou de escalonar as intervenções colocadas em prática e de observar os efeitos destas mudanças sobre os indicadores escolhidos ${ }^{5}$.

\section{A engenharia cooperativa}

No nosso último livro (LAMEUL; LOISY, 2014, p. 199), nos interessamos pela natureza dos três modos de intervenção (pesquisa, ação e formação) e à maneira como eles se posicionam uns em relação

5 Escrito a partir do relatório coletivo Hy Sup. 
aos outros. Nós destacamos como seu alinhamento ou imbricação configuram as articulações diferentes entre os autores, questionamos os métodos utilizados para favorecer o encontro da pesquisa e da ação, assim como o ganho atual dos estudos. A engenharia cooperativa faz parte das tendências metodológicas que descobrimos entre as que são cada vez mais mencionadas ou utilizadas no campo da pesquisa. Como a design-based research, a engenharia cooperativa coloca-se na categoria das análises centradas no utilizador e, de maneira geral, pertencem à família das metodologias nas quais os usos estão no centro da concepção dos dispositivos que lhe são destinados.

A engenharia cooperativa oferece, por exemplo, a possibilidade de acessar uma fina escolha de conhecimentos que permitem distinguir o que pode ser considerado como sendo muito específico da situação e o que pode ser considerado como genérico e suscetível de fornecer indícios de acesso à transposição. Para Sensevy (2011, p. 678), as engenharias cooperativas se baseiam na "determinação comum de fins comuns" entre professores e pesquisadores. Estes fins comuns se instituem no trabalho coletivo do saber sobre quatro dimensões, sobre a identificação de elementos importantes da prática do saber, sobre a maneira como vão ser escolhidos os elementos essenciais, sobre os dispositivos usados para atualizá-los, sobre as formas que vão dar nas estratégias e nos gestos utilizados no ensino".

Para ilustrar a realidade das engenharias cooperativas, é interessante mencionar os lugares de educação associados (LéA) desenvolvidos a partir de 2011 pelo Instituto Francês de Educação IIFE-ENS de Lyon, pois trata-se de um dispositivo verdadeiramente original, suscetível de criar um quadro a partir do qual possa ser utilizada a engenharia cooperativa. Considerando a educação como um fato social total (Mauss, 1994) onde se inserem as dinâmicas da vida social dentro das interações múltiplas entre os diversos atores, os LéA associam pesquisadores e profissionais em relação aos projetos de pesquisa baseados nas ações concretas da educação.

Postulando que a educação depende, sobretudo, do contexto no qual está inserida e que está submetida a influências complexas, a pluralidade de atores da LéA de uma parte trabalha para a tomada em consideração da complexidade local, de outra parte estabelece como objetivo, desde o início, integrar a difusão dos resultados das pesquisas e das fontes que elas produzem em direção aos atores da 
educação envolvidos no mesmo questionamento e, mais geralmente, na direção da formação. [...] (LAMEUL; LOISY, 2014, p. 208).

Por sua vez, as engenharias cooperativas implantadas no seio das LéA parecem entreter uma real proximidade com as démarches dispositives, mais particularmente estudadas aqui e que nós detalharemos na sequência.

\section{Precisão em torno do conceito de 'démarche dispositive'}

De acordo com Geneviève Jacquinot e Hugues Choplin (2002, p. 187),

a démarche dispositive, híbrida por natureza, se propõe a dar conta das lógicas de uso e das interações entre os elementos heterogêneos que intervêm no processo de colocar em prática o dispositivo, ele mesmo inovador. Na hipótese construída no quadro de uma démarche dispositive, o dispositivo da formação pode tomar o lugar do objeto negociável no centro do processo de inovação pedagógica. $\mathrm{Na}$ articulação do dispositivo e da inovação, a caminhada não permite apenas pensar de outro jeito os sistemas de ensino e aprendizagem (sobretudo informatizada), mas questiona também a ligação que se dá entre a inovação pedagógica e a transformação da atividade da pesquisa. [...]

Seu valor heurístico propõe um lugar (espaço) e um processo (tempo), seja um "objeto comum" para negociar com e entre os sujeitos (social). É todo um jogo de dinâmicas que se vê questionado: dimensão dinâmica daquilo que se faz no dia a dia; dimensão inovadora na atualização de práticas novas a instituir; dimensão semiótica contribuindo para a explicitação do sentido desta novidade em relação às práticas antigas tão firmadas e tradicionais que parecem "naturais".

$\mathrm{Na}$ perspectiva de esclarecer o processo de cumulatividade dos resultados da pesquisa mencionada, esta noção emergente pode ser comparada com outros trabalhos relativos ao dispositivo: por exemplo, os de Monique Linard (1989) que definem o dispositivo como um agenciamento estratégico e finalizado de fontes humanas e materiais; os de Brigitte Albero $(1989,2000)$ que se inspiram e detalham o "dispositivo" como uma comunicação emergente de fontes, de in- 
terações e de intenções de atores, ao mesmo tempo heterogêneo e evolutivo no tempo, constituindo as dimensões ternárias (ideal, funcional de referência, vivido) que ele conceitualizou em seguida (ALBERO, 2010, 2011); aqueles, enfim, de Daniel Peraya e Bernadette Charlier (2002) e os de Didier Paquelin (2009) etc. A constatação de uma tal diversidade e riqueza de proposições não deve ser lida como um convite para colocar em perspectiva estas diferentes definições de uma mesma noção de "dispositivo"? Não significaria uma maneira de especificar as características sob o olhar dos contextos e de otimizar, assim, os meios de análise dos dispositivos inovadores, cada vez mais complexos aos quais estamos confrontados?

Para melhor destacar uma noção que a sociedade contemporânea parece ter cada vez mais necessidade, tentarei colocar em perspectiva a noção essencial de démarche dispositive em relação às análises citadas que se inscrevem no mesmo sentido. Este exercício tem a finalidade de reforçar a visibilidade, de sublinhar as especificidades de cada método para melhor desempenhar sua complementariedade.

Tabela 2: Características da démarche dispositive em relação a outras 'démarches' (design based research, engenharia cooperativa)

\begin{tabular}{|l|l|l|l|l|}
\hline Itens & Especificidade/originalidade & $\begin{array}{l}\text { Autores de } \\
\text { referência }\end{array}$ & $\begin{array}{l}\text { Situação pertinente a } \\
\text { estudar }\end{array}$ & $\begin{array}{l}\text { Postura do } \\
\text { pesquisador }\end{array}$ \\
\hline $\begin{array}{l}\text { Démarche } \\
\text { dispositive }\end{array}$ & Um objeto comum a negociar & $\begin{array}{l}\text { Jacquinot } \\
\text { e Choplin } \\
\text { (2002) }\end{array}$ & $\begin{array}{l}\text { Mudança, } \\
\text { Inovação }\end{array}$ & $\begin{array}{l}\text { Semi-exte- } \\
\text { riorização. } \\
\text { Coanálise, } \\
\text { coprodução }\end{array}$ \\
\hline $\begin{array}{l}\text { Design based } \\
\text { research }\end{array}$ & $\begin{array}{l}\text { Trabalho participativo, intera- } \\
\text { tivo, centrado no uso da teoria }\end{array}$ & $\begin{array}{l}\text { Van den } \\
\text { Akker et al. } \\
(2006)\end{array}$ & $\begin{array}{l}\text { Estudo de caso no } \\
\text { tempo }\end{array}$ & Cooperação \\
\hline $\begin{array}{l}\text { Engenharia } \\
\text { cooperativa }\end{array}$ & $\begin{array}{l}\text { Determinação comum de fins } \\
\text { comuns. Trabalho coletivo do } \\
\text { saber }\end{array}$ & $\begin{array}{l}\text { Sensevy } \\
(2011)\end{array}$ & $\begin{array}{l}\text { Pesquisa de compre- } \\
\text { ensão de processos. } \\
\text { Distinção do que é } \\
\text { especíico e genérico } \\
\text { na situação }\end{array}$ & Colaboração \\
\hline
\end{tabular}

Este quadro recapitulativo de algumas das práticas de pesquisa articuladas à ação faz aparecer um grande número de pontos comuns entre os diferentes métodos (centralizar sobre a atividade humana, 
colaboração entre os diversos atores, acoplamento dos dispositivos de pesquisa e de formação). Ele destaca, tanto a diversidade dos objetos principais ao redor dos quais se organiza a pesquisa (dispositivo, estudo de caso longitudinal, saber) e as nuances em termos de postura dos atores. É isso que questionaremos em seguida.

\section{As posturas dos pesquisadores e dos profissionais em evolução}

Por diferentes razões, a démarche dispositive pode parecer bem complexa para os atores. De um lado, a fim de deixar livre a iniciativa para o surgimento do jogo da inovação, a postura dos autores deve integrar uma distância qualificada de semi-exterioridade em relação ao dispositivo inovador por si mesmo. De outro lado, se falarmos dos terrenos estudados por Jacquinot e Fichez (2008), percebemos que este estudo foi feito levando em conta sua evolução progressiva, tanto sob a influência interna própria à dinâmica de uma experiência pedagógica já antiga e, sob a pressão externa tanto nacional como internacional desejada pelas autoridades políticas.

Pesquisadores e profissionais (responsáveis políticos, professores ou engenheiros dedicados à pesquisa) estão permanentemente sob estes movimentos tensos. A pesquisa deve, portanto, continuamente tentar "ultrapassar os limites do ponto de vista objetivo e, tendo que escolher as práticas de fora como fato dado, em vez de construir o princípio gerador situando-se dentro do movimento de sua efetivação". Encontramos, neste caso, uma proximidade forte com o que define a caminhada praxeológica, que, segundo Bourdieu (1972, p. 163), tem por objeto

não somente as relações objetivas, mas as relações dialéticas entre estas estruturas objetivas e as disposições estruturadas nas quais se atualizam e que tendem a reproduzir, isto é, o processo duplo de interiorização da exterioridade e de exteriorização da interioridade. [...]

Quando estudamos a articulação entre dispositivo e disposição (LAMEUL; JEZEGOU; TROLLAT, 2008), buscando encontrar as articulações possíveis entre os elementos do dispositivo proposto e as disposições próprias a cada um que aprende, estávamos provavelmente muito próximos desta questão. E isso nos conforta e nos deixa a vontade para trabalhar e refletir com os atores quando tomam suas posições. 
De maneira mais global, os métodos de pesquisa aos quais nos interessamos contribuem para assentar as novas configurações onde o desenvolvimento profissional do professor ou de seu acompanhante (engenheiro pedagógico ou conselheiro) pode se fazer na participação da caminhada da pesquisa. A conjugação do dispositivo de pesquisa com os dispositivos de ação e de formação constrói um quadro tanto mais interessante para produzir um conhecimento o mais próximo possível da ação humana, onde as posturas dos atores são precisas e conscientes. Como falou Brigitte Albero,

esta contribuição reflexiva da pesquisa à ação anda sozinha, desde que se trate de abandonar as concepções aplicacionistas de uma expertise consolidada, para inventar os esquemas e os modelos de um acompanhamento mais partilhado do desenvolvimento individual e coletivo. [...] (2013, p. 96).

O equilíbrio entre as produções de diferentes naturezas (produção de conhecimento, desenvolvimento humano, eficácia da ação) está, sem dúvida, correlacionado ao equilíbrio que cada ator saberá ter no seio de sua postura pessoal entre suas intenções, seus valores e suas ações, assim como na harmonia dos equilíbrios entre as posturas dos diferentes atores. A caminhada dispositiva que focaliza sobre o espaço de negociação no centro do dispositivo poderá constituir um destes métodos suscetíveis de conciliar as duas exigências de uma situação inovadora: manter o título das diversas produções, combinando pesquisa e ação e saber adotar as posturas mais adequadas para jogar pertinentemente sobre os diferentes registros de intervenção, da pesquisa e da formação.

\section{REFERÊNCIAS}

ALBERO, B; BRASSAC, C. Revue française de pédagogie. In: Une approche praxéologique de la connaissance dans le domaine de la formation. Éléments pour un cadre théorique, 2013.

ALBERO, B. L'analyse de l'activité en sciences de l'éducation : entre aspirations scientifiques et exigences pragmatiques. In: Travail et apprentissages, 2013. 
ALBERO, B. Approche trilogique des dispositifs en formation : pourquoi est-ce que les choses ne fonctionnent-elles jamais comme prévu ? In: Gaux C. et Vinatier I. (org.), Outils pour la formation, léducation et la prévention : contributions de la psychologie et des sciences de léducation. Nantes, Actes du colloque OUFOREP, 2011.

ALBERO B. La formation en tant que dispositif : du terme au concept. In: Charlier B. et Henri F. (org.), La Technologie de léducation : recherches, pratiques et perspectives, Paris, Presses universitaires de France, 2010.

ALBERO, B; LINARD, M.; ROBIN, J.-Y. Petite Fabrique de l'innovation à l'université. Quatre parcours de pionniers, Paris, L'Harmattan, 2009.

ALBERO, B. L'Autoformation en contexte institutionnel : du paradigme de l'instruction au paradigme de l'autonomie. Paris, L'Harmattan, coll. Éducation et formation, série Références, 2000.

BOURDIEU, P. Esquisse d'une théorie de la pratique, Genève, Droz, 1972.

DESCHEREYVER, N.; CHARLIER, B. (org.) (2012), Dispositifs hybrides. Nouvelles perspectives pour une pédagogie renouvelée de l'enseignement supérieur. Rapport final, 2012. Acessado em 14 outubro 2016: http://prac-hysup. univ-lyon1.fr/spiral-files/download?mode $=$ inline $\&$ data $=1757974$

GUÉRIN, J. Activité collective et apprentissage : de l'ergonomie à l'écologie des situations de formation. Paris, L'Harmattan, coll. Action Savoir, série Recherche, 2012.

DELAUNAY, G; JACQUINOT, G; MONNOYER, L. (org.). Le Dispositif. Entre usage et concept, Hermès, 1999.

JAQUINOT, G.; FICHEZ É. L'Université et les TIC. Chronique d'une innovation annoncée. Bruxelles, De Boeck Supérieur, coll. Perspectives en éducation et formation , 2008.

JACQUINOT, G; CHOPLIN, H. Éducation permanente. In: La démarche dispositive au risque de l'innovation, 2002.

LAMEUL, G.; SIMONIEN, S.; ENEAU, J.; CARRAUD, F. Regards croisés de chercheurs praticiens sur le dispositif de formation hybride FORSE : comment les enseignants transforment-ils leur modèle pédagogique en intervenant en ligne ? In: International Journal of Technologies in Higher Éducation, 2011.

LAMEUL, G.; JÉZÉGOU, A; TROLLAT, A-F. (org.) Articuler dispositifs de formation et dispositions de l'apprenant, Lyon, Éditions Chronique sociale, 2009. 
LAMEUL, G; LOISY, C. (cood.). La Pédagogie universitaire à l'heure du numérique : questionnements et éclairages de la recherche, Bruxelles, Éditions De Boeck, 2014.

PAQUELIN, D. LAppropriation des dispositifs numériques de formation : du prescrit aux usages. Paris, L'Harmattan, 2009.

PERAYA, D.; CHARLIER, B. Technologie et innovation en pédagogie.

Dispositifs innovants de formation pour l'enseignement supérieur, Bruxelles, Éditions De Boeck, 2002.

REMOUSSENARD, P. Les théories de l'activité entre travail et formation. Savoirs, 2005.

SENSEVY, G. Le Sens du savoir. Éléments pour une théorie de l'action conjointe en didactique. Bruxelles, Éditions De Boeck, coll. Perspectives en éducation et formation, 2011.

VAN DEN AKKER, J.; GRAVEMEIJER, K.; MCKENNEY, S.; NIEVEEN, N. Educational design research. London, Routledge, 2006. 